\title{
Fighting counterfeiting at the nanoscale
}

\author{
Exploring the inherent complexity and uniqueness of nanostructures may provide the effective solution for \\ security applications.
}

$\mathrm{T}$ he threat to the world economy, national security and human health associated with counterfeiting of products and intellectual property infringement has been on a particularly alarming increase in recent years. Globalization and the growth of the Internet are the main culprits most commonly blamed for the rapid rise of the trade volumes in counterfeited and pirated products, which currently amount to nearly $10 \%$ of all goods sold worldwide.

Ironically, for all its high-tech advances, the semiconductor industry has been extremely vulnerable to counterfeit electronics. In fact, fake components can now be cloned so precisely that it is nearly impossible to distinguish them from the genuine article. Moreover, despite a wealth of available anticounterfeiting techniques such as holograms, tamper-evident closures, tags, markings and radiofrequency identification labels, most of them, however costly and sophisticated, can be copied within 18 months. Therefore, with no silver bullet solution for counterfeiting in hand, the development of new approaches and improvement of current technologies is of urgent need.

To this end, nanotechnology might be able to offer a helping hand in preventing illicit copying of products and intellectual property by providing highly secure anti-counterfeiting methods that rely, for example, on naturally occurring physical parameter variations at the nanoscale, or the fabrication of covert nanofeatures completely invisible to the eye.

Particularly in optical security devices such as holograms, nanopatterning techniques can assist in providing an enhanced level of security by making them easy to verify but challenging to forge. Likewise, encoded nanostructures, nanoparticles or molecular self-assemblies have been successfully used for nanobarcoding to create nanoscale dataencryption keys.

In laser surface authentication applications, which are commonly employed in biometrics for documents and goods, the identification process involves optical inspection of the surface roughness of an object. The enhanced complexity and uniqueness of the nanoscale surface features are virtually impossible to modify in a controllable manner, making these 'fingerprints' immune to attacks. Another potent identification tool is quantum dot (QD) tagging, which is commonly used

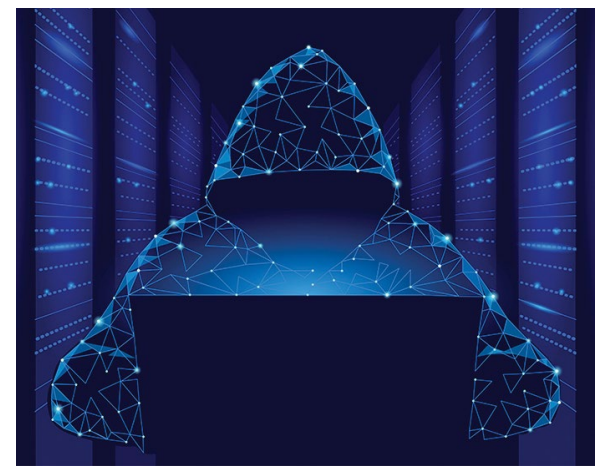

that behaves indistinguishably from the original PUF.

Nanoscale electronic devices, once again, could offer the possibility of addressing these security shortcomings. At least, as far as the nanotechnology community is concerned, there is no lack of ideas. One potential solution is to use variations in resistive states of memristors in a standard nanocrossbar architecture to fabricate on-chip memristive device-based PUFs. However, for this approach to generate highquality randomness, the device set voltage and bias duration have to be precisely controlled, which remains challenging.

Taking advantage of intrinsic cell-

in bioanalytical sciences and bioengineering for optical coding of biomolecules. This approach is based on the optical properties of semiconductor QDs exhibiting a spectral response that can be readily controlled by varying the QD size or density, which serves as a tuning parameter for the intensity of the read optical signal. Much like QD tagging, the surface-enhanced Raman scattering (SERS)-fluorescence technique exploits unique electromagnetic spectra of metal nanoparticles to produce secure nanoscale tags.

Among numerous examples of potent methods used for authentication and identification applications, as well as cryptographic key generation, one that stands out in particular is physically unclonable functions (PUFs). Although not originally designed for electronics, these security primitives have found widespread applications in hardware security, serving as a unique identity for electronic devices such as integrated circuits, radiofrequency identification tags and field-programmable gate arrays. For fortifying electronic devices, conventional microelectronic circuit-based PUFs, such as ring oscillator PUFs, arbiter PUFs and SRAM PUFs, exploit uncontrollable minute process variations in traditional complementary metal-oxide-semiconductor fabrication technology. Rather than using digital memory to store secret information or requesting a user to provide it, it evolves as a result of a physical characteristic of the system, which presumably cannot be cloned. Yet multiple studies have revealed how machine learning techniques can create attacks to break various types of PUFs through constructing a computer algorithm to-cell and cycle-to-cycle programming memory nanodevices provides another way of creating virtually unclonable and reconfigurable PUFs. Carbon nanotube (CNT)-based PUFs have also been reported, which use unique physical characteristics of CNT field-effect transistors or take advantage of inherent imperfections of CNTs to create self-assembled CNT primitives. Two-dimensional materials, such as transition metal dichalcogenides and graphene, are another class of nanomaterials potentially suitable for anti-counterfeiting technologies. Some of the recent studies show that vacancies, impurities, grain boundaries and other structural defects that occur, for example, during the growth process, create spatially varying nanostructures with a unique optical response that can be leveraged to construct cryptographic primitives.

Exploring nanoscale phenomena for of how nanotechnology rooted in wellestablished microscale concepts can expand on these ideas to promote the existing technology to an entirely new level, offering positive societal and economic implications. However, any continued progress in emerging nanotechnologies for anti-counterfeiting and their eventual implementation will inevitably require further experimental validation, performance evaluation and reliability assessment of the nanoscale devices. These efforts are already underway.

Published online: 5 June 2019 https://doi.org/10.1038/s41565-019-0484-0 variability at the nanoscale of phase-change security applications is a notable example 